\title{
TRANSMUTATION OF RADIOACTIVE WASTE
}

\author{
V.Chulikov, A. Pauls, V. Savchenko \\ Linguistic Advisor: Teacher A.V. Tsepilova \\ Tomsk Polytechnic University, Russia, Tomsk, Lenin str., 30, 634050 \\ E-mail: vladimir666c@gmail.com
}

Abstract

The present work is devoted to the problem of radioactive waste transmutation, i.e. transformation of longlived and toxic radionuclides into less toxic isotopes with shorter half-lives. While storage and burial cannot completely prevent radioactivity from getting into the environment, transmutation is a potential way to resolve the problem of radioactive waste. Different types of nuclear transmutation taking place under different conditions are described and analyzed. They include transmutation of long-lived fission products in a neutron field, generation of neutrons for providing transmutation of long-lived fission products, electronuclear method of neutron generation and controlled nuclear fusion. A review of literature devoted to the above-mentioned techniques is done.

Nuclear power is an important developing power source for the future. It represents a pure and effective energy resource. One of pressing problems of nuclear power is the problem of increase of safety of a postreactor fuel cycle where the important place is taken by the problem of dealing with radioactive waste (NW). Storage and burial cannot completely prevent radioactivity from getting into the environment. Only the transmutation can resolve the problem of radioactive waste disposal. Transmutation means transformation of long-lived and toxic radionuclides into less toxic substances with shorter half-lives. The idea is that the program of a transmutation should transform the problem of long-term isolation of NW to the problem waste storage during several tens or hundreds years.

It is known that NW appears at various stages of production: from uranium mining to processing of spent fuel. The NW which appears as a result of spent fuel processing is the most difficult to dispose of. It contains the major part of fission products characterized by high activity, thermal emission and a long period of recession of activity. A characteristic feature of radioactivity is that it can neither be destroyed nor neutralized by chemical methods. It is possible to destroy radioactivity only by nuclear reactions, transferring radioactive isotopes to stable, long-lived or short-lived isotopes, or by destroying the nucleus. Transmutation of all long-lived radionuclides can be considered the most important task. The role of transmutation of long-lived actinides and, to a lesser extent, of the fission products accumulating in reactors, becomes more and more significant in development of fuel cycles, both for modern reactors, and for future nuclear power systems. It will allow to reduce the load on the places of radioactive waste burial or to eliminate the problem of "eternal" burial of the most dangerous long-lived radionuclides.

First of all, let us consider the transmutation of long-lived fission products in a neutron field.

Long-lived radionuclides with high specific activity, which appear when processing spent fuel of nuclear reactors, pose the greatest danger. It is also necessary to take into account the fact that similar highly active waste can appear when processing the spent fuel of electronuclear and thermonuclear facilities with a 
subcritical blanket, which are usually called hybrid thermonuclear reactors.

The speed of a transmutation of long-lived fission products in a neutron field does not depend on time and depends on neutron flux density, so with its increase the speed of reduction of nuclide amount also increases. The rate of this recession is characterized by average lifetime of radionuclide in a neutron field. If the neutron flux density is equal to zero, average lifetime of this radionuclide is equal to the half-life period of this nuclide. If If the neutron flux density is not equal to zero, average time lifetime of radionuclide is called the effective half-life period of the radionuclide which is in a neutron field. The nature of nuclide amount change in time will depend on entry conditions, i.e. on the moment when transmutation process begins. If the transmutation begins at the moment when in a nuclear system there is such an amount of radionuclide that corresponds to equilibrium value, the time of transmutational process is reduced. The development of such transmutation technique would allow to reach equilibrium state quickly enough. The most important indicators of the efficiency of neutralization of long-lived NW are as follows: average lifetime of the transmuted radionuclide in a neutron field, inevitable losses of radionuclides in a production cycle of transmutation, generation of excess neutrons for transmutation, safety of the transmutational cycle for environment. [1]

The next technique is generation of neutrons for providing transmutation of long-lived fission products. It is necessary to considercritical systems (nuclear reactors).

The critical system is characterized by neutron balance of the self-sustaining chain reaction of fission. It is possible to release neutrons for a transmutation only as a result of redistribution of neutron balance in a chain reaction. If in the future nuclear power is based mainly on fast reactors, secondary fuel will be needed only for replenishing of burning primary fuel, and also for ensuring the development of a nuclear power supply system. Then in fast reactors the part of uranium in screens can be replaced by transmuted long-lived radionuclides, i.e. such a reactor can be considered to be a source of neutrons for transmutation. Transmutational potential of such fast reactor (in case it provides fuel for itself) could make $\sim 0,5$ neutrons per one act of fission. However, transmutational potential cannot be characterized only by the number of generated excess neutrons. For effective transmutation rather high density neutron flux density is also required. Otherwise, we would have to wait for equilibrium transmutation for centuries. As level of neuron flux in the screen of a fast reactor is connected with flux level in reactor core, the increase in neutron flux density will cause the need to increase the density of energy release in the core, which has restrictions on heathydraulics, operability of fuel elements and safety. [2]

Let us now say a few words about the electronuclear method of neutron generation (non- reactor systems).

Electronuclear method of neutron generation is based on the use of nuclear fission reactions of nuclei of heavy elements $(\mathrm{Pb}, \mathrm{Tb}$ and others), accelerated to high energies (1-1,5 GeV) by protons. The process of interaction of high-energy particles with nuclei has a cascading nature. When a beam of accelerated particles is absorbed by the target, fission reactions with subsequent cascade-evaporation multiplication of neutrons are induced in it. Neutron yield (average number of neutrons emitted from the target per one bombarding 
particle) is proportional to the value of the kinetic energy of bombarding particles at the moment of the first inelastic collisions with nuclei of the target. The value of the neutron yield as a result of the cascading process also depends on the target material and its size. Due to the permanently leakage of neutrons and parasitic absorption in construction materials, coolant and moderator lose about $10 \%$ of the generated neutrons. The number of neutrons produced in ENI for the needs of transmutation is 0.8 neutrons per fission act. These are additional neutrons in relation to the neutron balance of nuclear energy systems that can be used for transmutation of long-lived NW power reactors. Not all of the additional neutrons can be used for transmutation of long-lived radionuclides because fission energy was used for their obtaining (for power accelerator), which caused the emergence of fission products.

One more technique that could be used as a source of neutrons for transmutations is controlled nuclear fusion.

Controlled nuclear fusion of heavy isotopes of hydrogen is traditionally considered as a power source with almost inexhaustible fuel base. Researches in the field of NF, which have been conducted for nearly half a century, are now at such a stage that the moment of obtaining intensive controlled thermonuclear reaction is quite close.

The simplest way of implementation of thermonuclear reaction is synthesis of deuterium + tritium (fuel $\mathrm{D}-\mathrm{T}$ ) with a positive power output, thus it is accompanied by the emergence of a fast neutron which carries away about $80 \%$ of the released energy into the blanket. However, higher plasma temperature is needed to induce a $(\mathrm{D}-\mathrm{D})$ reaction. Replenishing of a burnt deuterium $\left({ }_{1}^{2} D\right)$ doesn't make a problem since its stocks on our planet are almost inexhaustible $\left(0,014 \%\right.$ in natural water), and radioactive tritium $\left({ }_{1}^{3} T\right)$ with $\left(\mathrm{T}_{1 / 2}=12.3\right.$ years $)$. Taking into account inevitable losses of neutrons in a blanket due to "parasitic" absorption and leakage of radioactive decay, 1,06 nuclei of tritium is required for one (D - T) - reaction.

Along with $(\mathrm{D}-\mathrm{T})$ reaction, reaction between deuterium nuclei (the $\mathrm{D}-\mathrm{D}$, monofuel) which proceeds in two channels with approximately identical probability is also considered. Here one neutron and one tritium nucleus appear in on the average two (D-D) - reactions. The tritium nucleus can enter $(\mathrm{D}-\mathrm{T})$ reaction with generation of one more neutron. However, for inducing a (D-D) - reaction a higher temperature of plasma is needed. At the same time in this case it is not required to spend neutrons on tritium production.

An important advantage of a thermonuclear source of neutrons is rather low fraction of thermonuclear capacities which need to be included into a nuclear power supply system. If, for example $\eta_{\text {тр }}=0,5$ neut./fission act is required for transmutation of long-lived products, then the share of thermonuclear capacities will make about $15 \%$ of power of the whole system. If an electronuclear installation (ENI) is used for this purpose, the share of capacities connected with ENI (provision of energy for accelerators), makes more than $60 \%$.

Generation of neutrons in partially catalyzed (DDT) - synthesis is also considered. 0,5 neutrons with $\mathrm{Ev}=2,45 \mathrm{MEV}$ and 0,5 neutrons with $\mathrm{Ev}=14,1 \mathrm{MEV}$ will be generated for one reaction due to combustion of tritium formed in the reaction. The rigid component of this neutron source can be multiplied by placing 
lead or beryllium behind the first wall. The exit of neutrons for one partially catalyzed (DDT) - reaction (taking into account generation of neutrons in accompanying (D-D) - reaction) will make 1,20.

Although it is less than the neutron output in (D-D) - reactions taking into account reproduction, there is no need to spend neutrons on tritium reproduction. Therefore, if we assume that parasitic absorption is 0,25 , then 0.95 neutrons per one partially catalyzed (DDT) - reaction may be used for transmutation. This size is three times greater, than the corresponding generation of neutrons for transmutation by means of (DD) - synthesis reaction. [3]

\section{CONCLUSION}

Due to the large-scale development of nuclear power the problem of processing NW, and its further use became important. In scientific publications various versions of physical solutions concerning implementation of long-lived NW transmutation in both nuclear reactors, and in nuclear installations which haven't been practically implemented (electronuclear and thermonuclear installations) are discussed. Researches on NW transmutation physics will allow to formulate at least conceptual technical requirements to such installations.

Now it can be clearly understood that it is impossible to develop nuclear power of the future with old types of nuclear reactors. It is necessary to develop technologies and designs, such as subcritical nuclear reactors operated by accelerators of charged particles. These systems will allow to eliminate the use of enriched uranium and plutonium and to completely solve the problem of spent nuclear fuel and radioactive waste, to provide non-proliferation of dangerous nuclear materials.

\section{REFERENCES}

1. E.O. Adamov, I.H. Ganev, A.V. Lopatkin, V. G. Muratov, V. V. Orlov. (1999). Transmutational fuel cycle in large-scale nuclear power industry of Russia;

2. G. M. Pshakin, N. I. Geraskin, V.A. Apse (2004). Nuclear non-proliferation;

3. V.A. Apse, A.E. Sintsov, A.N. Shmelyov, G.G. Kulikov, V. V. Artisyuk (2001). Radioactive waste: neutralization ways;

4. V.A. Apse, A.N. Shmelyov (2006). Bases of safe handling of radioactive waste;

5. «Development of nuclear power and integrated power systems»:

http://energetika.in.ua/ru/books/book-4/part-1/section-8/8-1 